\title{
Optically Driven Quantum Computing Devices Based on Semiconductor Quantum Dots
}

\author{
Xiaoqin Li, ${ }^{1}$ Duncan Steel, ${ }^{1,4}$ Daniel Gammon ${ }^{2}$ \\ and L. J. Sham ${ }^{3}$
}

Received February 10, 2004; accepted April 12, 2004

\begin{abstract}
This paper concerns optically driven quantum logic devices based on semiconductor quantum dots. It provides a brief review of recent theoretical and experimental progress towards building such devices and a description of a possible direction of further research. We consider both the exciton and the electron spin as a potential qubit. Quantum dot fabrication and single dot spectroscopy studies are briefly discussed followed by a description of experimental demonstrations of basic quantum logic operations. A scheme for a scalable quantum computer based on optical control of electron spins localized in quantum dots is described in detail. Important lessons as well as challenges for future research are summarized.
\end{abstract}

KEY WORDS: Quantum computing; quantum entanglement; semiconductor quantum dots; ultrafast optical spectroscopy.

PACS: 78.67.Hc; 42.50.Md; 03.67.Lx; 42.50.Hz.

\section{INTRODUCTION}

Semiconductor quantum dot (QD) studies have evolved from ensembles to single and coupled dots, opening up the possibility of building quantum devices based on the existing infrastructure for quantum optoelectronic semiconductor fabrication. It is believed that these dots may be used as the basic building blocks for future quantum information processing devices. The focus of this paper is on building optically driven quantum logic devices based on semiconductor QDs. We aim to provide a simple

\footnotetext{
${ }^{1}$ FOCUS, Harrison M. Randall Laboratory of Physics, The University of Michigan, Ann Arbor, MI 48109-1120.

${ }^{2}$ Naval Research Laboratory, Washington, DC 20375-5347.

${ }^{3}$ Department of Physics, University of California, San Diego, La Jolla, CA 92093-0319.

${ }^{4}$ To whom correspondence should be addressed. E-mail: dst@eecs.umich.edu
} 
review of the rapid theoretical and experimental progress made in the past few years and discuss the future directions.

Following the first theoretical proposal ${ }^{(1)}$ to use optical excitation in confined semiconductor systems for quantum computation, early optical spectroscopy studies of single quantum dots demonstrated the existence of isolated optical excitations ${ }^{(2,3)}$ and provided the promising prospect that such localized excitations may be controlled individually and used as carriers of quantum information. ${ }^{(4)}$ More detailed exciton-based proposals of implementation followed. ${ }^{(5-7)}$ Following studies of the coherent nonlinear optical response ${ }^{(8)}$ and coherent optical control ${ }^{(9)}$ at the single dot level, an experimental breakthrough was made in 2001 when several groups demonstrated Rabi oscillations of excitons confined in single quantum dots using a variety of optical techniques. ${ }^{(10-13)}$ Rabi oscillation of a qubit is an essential forerunner to arbitrary single qubit operations.

The essence of quantum computation and information processing lies in the generation of entanglement of qubits. Theoretical studies show that, while not scalable, two excitons in a single dot can be used to demonstrate simple quantum algorithms such as the Deutsch-Jozsa algorithm with pulse-shaping techniques for quantum operations. ${ }^{(7)}$ Quantum coherence between arbitrary states within the computation basis was shown to persist for the lifetime of dipole transitions. ${ }^{(14,15)}$ Entangled states of two polarized exciton states in a single dot were created and detected optically. ${ }^{(14,16)}$ A key experimental demonstration of the capability of coherent control of excitons for conditional quantum operations is the demonstration of a simple two-bit quantum gate based on two excitons confined in a single quantum dot. ${ }^{(17)}$

A proposal for a scalable system is based on using single electron spins, with much longer coherence times than the above systems based on excitons, residing in closely spaced quantum dots with gate control of spins in separate dots. ${ }^{(18)}$ There is considerable experimental effort currently directed at developing appropriate gate control of the spins. Optically driven electron spins confined in QDs is a promising alternative to electronic control for future quantum information processing since the speed for optical operations can easily exceed a THz. ${ }^{(19,20)}$ The recent demonstration of fabrication of structures, where one extra electron is confined in a single dot, ${ }^{(21)}$ has been a promising step towards achieving these proposals.

For charged quantum dots of the above type, optical excitation creates a charged exciton (trion), which can be used to rotate the electron spin. (22) This charged exciton state thus serves as an auxiliary state that provides optical access to the electron spin. Currently, the properties of negatively charged dots and, in particular, of trions are being studied intensely. ${ }^{(23)}$ 
In this review, we follow a particular framework for universal quantum computation ${ }^{(24)}$ of defining qubits, the single qubit operations and the two-qubit conditional operations on demand, initialization and readout. We discuss the experimental demonstrations achieved so far based on exciton qubits, and describe proposals for the next generation of systems based on manipulation of spin qubits.

\section{QUBITS IN SEMICONDUCTOR QUANTUM DOTS}

Optical excitation of an electron into the empty conduction band of a semiconductor leaves a hole in the valence band. The electron and hole attract each other via the Coulomb interaction and form a bound-state exciton. The quantum dot serves as a trap with an effective potential for the electron and one for the hole as shown in Fig. 1(a). Because the band gap forms an excitation barrier, the effect of the Coulomb interaction of the large number of electrons in the ground state with the excited electron simply renormalizes its attraction with the hole by a dielectric constant and its mass in combination with the influence of the crystal potential.Unless there are too many excited electrons, the electron-hole pair
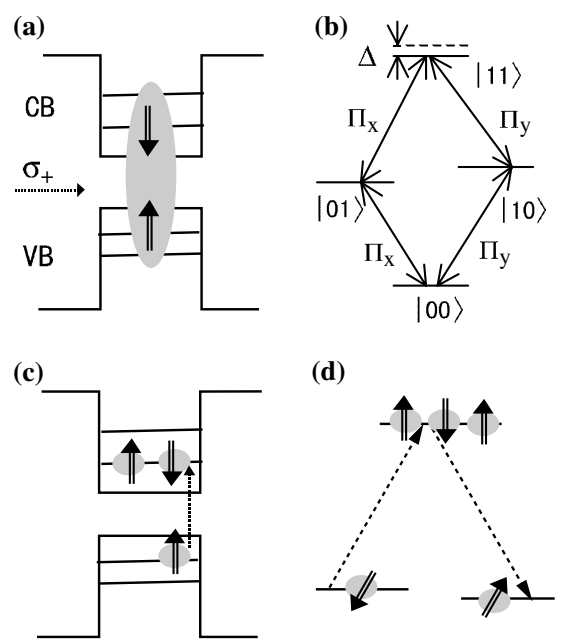

Fig. 1. Optical control of exciton-based qubits and spin-based qubits. (a) The single particle energy levels in a neutral dot indicating the optical excitation of an exciton qubit; (b) the excitation diagram of a two-exciton system confined in a single dot; (c) the single particle energy levels in a dot indicating the electron spin and the optically controlled exciton; (d) spin manipulation through the Raman process of the two lowest spin states and the trion state. 
behaves remarkably like a positronium atom. The presence and absence of an exciton in a dot can serve as a qubit.

In an alternate scenario, one can dope each QD with a single electron. The spin of each localized electron then serves as a qubit. The state of the single electron spin is controlled via a stimulated Raman transition via the trion (electron-exciton) state ${ }^{(2)}$ as illustrated in Fig. 1(c) and (d).

Laser pulses on the order of tens of femtoseconds are readily available in laboratories nowadays. The interaction energy of the excitons and the Zeeman energy of the spin levels are both of the order of meV. To minimize unintended dynamics of the unselected states in either the exciton or the spin qubit system, the pulse width cannot be arbitrarily short. Nevertheless, optically driven logic devices can be operated at clock speeds approaching the $\mathrm{THz}$ regime. Pulse shaping schemes ${ }^{(7,22)}$ can be implemented to prevent the addition of errors in logical qubits. The clock speed of the operations is thus orders of magnitude faster than electrical or microwave control for the same spin qubit system.

Minimizing the errors in quantum operations caused by dephasing (and hence reducing the demand for error corrections) requires completing quantum operations before the system loses quantum coherence. The exciton coherence is limited by radiative recombination, whose time ranges from $100 \mathrm{ps}$ in the large interface fluctuation $\operatorname{dots}^{(8)}$ to $1 \mathrm{~ns}$ in the selfassembled dots. ${ }^{(25-27)}$ For operations of ps duration, the number of possible operations is $10^{2}-10^{3}$, which is currently believed to be insufficient for error correction. Shorter pulses are not viable, since the resultant increase in bandwidth results in excitation of other unintended states which compromises device performance.

The coherence time scale for electron spin based qubits is expected to be much longer, in the range of $1-100 \mu \mathrm{s}$. ${ }^{(28)}$ Electron spins make better qubits obviously from this perspective. Optical manipulation of a single electron spin is still a challenging task at this point. Initial demonstrations towards building quantum dot logic gates were based on excitons. However, such optical manipulation of exciton-based qubits is in fact a significant component of what is needed to optically control a single electron spin, and hence is the penultimate demonstration leading to an optically driven spin-based quantum logic device.

\section{FABRICATION OF QUANTUM DOTS AND SPECTROSCOPY STUDIES OF SINGLE DOTS}

Using advanced semiconductor fabrication technologies such as molecular beam epitaxy (MBE), coherent (defect free) islands spontaneously 
form on an initially flat 2-dimensional layer in the StranskiKrastanow growth mode of a highly strained system. Such a self-assembled mechanism is a rapid method to produce quantum dot arrays. Steady progress has been made in terms of fabricating quantum dots with more regular shape, size and positions. ${ }^{(29)}$ This type of quantum dot is believed to be promising for practical applications involving a large number of quantum dots and has been widely studied. Many challenges remain but already the properties of single pairs of vertically coupled dots have been explored optically. ${ }^{(30)}$ Other groups are attempting to develop laterally coupled dots, which may be necessary to scale to larger numbers of qubits. Considerable effort is needed, but researchers should eventually be able to provide designer QD molecules. For entanglement of two qubits from two dots on demand, it is better to have no interaction between two dots during quiescence but the two dots must be sufficiently close for the optical induction of qubit interaction such as via the optical RKKY interaction. $^{(19)}$

Another model system studied extensively is the natural quantum dot formed due to interface fluctuations in narrow quantum wells following growth interruption. The monolayer high islands provide the necessary quantum potential to localize spins and excitons. The most direct evidence of such localized excitons is provided by images taken by near-field optical microscopes. ${ }^{(31-33)}$ Besides near field microscopes, other approaches to achieve high spatial resolution include the use of shadow masks or mesas to limit the area of optical excitation. In this way single dot spectroscopy shows extremely sharp resonances. ${ }^{(2,3)}$ Such spectra can only be observed at the single dot level-ensemble measurements show broad energy spectra due to inhomogeneous broadening as a result of fluctuations in size and shape of the large number of dots.

Nonlinear spectroscopy measurements using continuous wave (CW) lasers have confirmed that pure dephasing processes are reduced as a result of reduced interaction between localized excitons. ${ }^{(8)}$ Furthermore, such CW spectroscopy studies have enabled detailed studies of biexciton formation in single dots, providing important information such as binding energy and nonradiative coherence times. ${ }^{(15)}$ Transient nonlinear studies on self-assembled QDs have turned out to be quite challenging and have required wave-guide structures to enhance the interaction length in initial studies. Such measurements have revealed long dephasing times of a few hundred picoseconds. ${ }^{(25,26)}$ In addition, optically induced entanglement of two orthogonally polarized exciton transitions in ensembles of InAs dots have been measured. ${ }^{(34)}$ 


\section{OPTICAL COHERENT MANIPULATION OF EXCITON QUBITS}

Universal computation may be achieved by quantum algorithms consisting of sequences made up from a set of arbitrary one-qubit operations and one entanglement creating two-qubit logic gate, such as a controlled-NOT (CNOT) gate or a square-root swap gate or a phase gate. A CNOT gate has now been demonstrated using two exciton qubits in a single quantum $\operatorname{dot}^{(17)}$ based on the demonstration of Rabi oscillations and production of entangled states. ${ }^{(16)}$

We note that a two-bit system in a single quantum dot forms the basis for the device where the necessary coupling is provided by the enhanced Coulomb interaction as a result of quantum confinement. Fig. 1(b) shows the excitation level diagram of such a two-exciton system. This simplest two-bit system involves the crystal ground state $(|00\rangle)$, two distinguishable excitonic states with orthogonal polarizations $(|01\rangle$ and $|10\rangle)$ and the biexciton state $(|11\rangle)$. It is important to note that the qubits are defined in the basis of the Bloch vectors of the exciton pseudo-spins. The value $1(0)$ corresponds to the presence (absence) of an exciton with the Bloch vector pointing up (down).

An important feature evident in the excitation picture is the large binding energy of the biexciton state due to the three-dimensional quantum confinement in QDs. The binding energy varies with the size of QDs, typically in the range of 3-4 meV. The large binding energy implies the following: the excitation of one exciton leads to a different excitation energy of the other exciton, shifting it down by an amount equal to the binding energy. This feature gives rise to the characteristic conditional dynamics needed for building a CNOT gate.

Biexcitons confined in a single quantum dot have been identified optically, and Rabi oscillations of the exciton to biexciton transition have also been demonstrated. ${ }^{(17)}$ An important achievement of such a demonstration is that the $\pi$ pulse can serve as the operational pulse of a CNOT gate. The performance of this exciton-based CNOT gate can be examined by comparing the physical truth table shown in Fig. 2 with the ideal one. Like a classical gate, the truth table provides the population of each state at the output corresponding to a particular input. As an example, if the input state of the system is 10 , after the gate operation, the populations in states $(00,01,10,11)$ are $(0.14,0.06,0.17,0.63)$ respectively, as opposed to the ideal $(0,0,0,1)$.

Quantum coherence and entanglement are critical to the superior performance of quantum logic devices compared to classical devices. The complete wavefunction immediately following a pulse that simultaneously excites both excitonic states can be written as $\psi=C_{0}|00\rangle+C_{+}|01\rangle+$ 


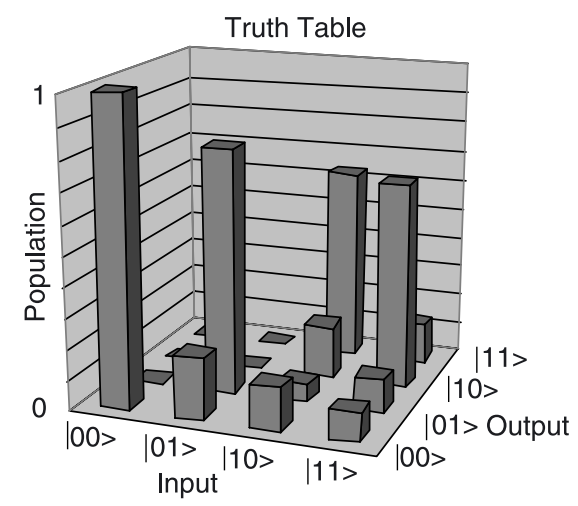

Fig. 2. Truth table of the numerically simulated CNOT gate using experimentally obtained parameters. In an ideal gate, the four highest bars are 1 and the others are 0 .

$C_{-}|10\rangle+C_{+-}|11\rangle$. The existence and duration of quantum coherence between arbitrary states in the computational basis was first measured in the weak excitation regime in a series of nonlinear spectroscopy studies using CW lasers. ${ }^{(8,14,15)}$ The creation of a specific entangled state requires laser pulses with pulse area (time-integrated electric field interaction) of $\sim \pi$. In an experiment aiming to create the Bell state $|01\rangle+|10\rangle$ using $\pi$ pulses coupled to both orthogonal excitons, coefficients were estimated to be $C_{0}=0.48, C_{+}=C_{-}=0.62, C_{+-}=0$, leading to entanglement entropy as high as $\sim 0.7$. The above discussion has assumed a pure quantum state following the optical excitation. Ref. 16 provides a more complete discussion of the mixed state entanglement. The temporal evolution of the non-radiative Raman coherence between states $|01\rangle$ and $|10\rangle$ was directly resolved in quantum beats measured in differential transmission (DT) geometry as shown in Fig. 3. The Raman coherence time was determined from the envelop decay of the beats, and was found limited by the lifetimes of the exciton transitions even in the strong field regime, thus showing that the potential coupling to other states under the high intensity optical field did not result in any detectable unintended dynamics or decoherence.

An extended definition of gate fidelity, ${ }^{(35)} \overline{\operatorname{Tr}\left[\rho_{P}(t) \rho_{I}(t)\right]}$, measures the overlap between the gate-produced mixed state denoted by the physical density matrix $r_{\mathrm{P}}$ and the ideal one $r_{\mathrm{I}}$, averaged over all possible initial states. Accounting for dephasing, the fidelity of the CNOT gate was calculated to be 0.7 assuming that the initial states can be prepared perfectly. ${ }^{(17)}$ An ideal gate would have fidelity of 1 while any real gate would have fidelity between 0 and 1 . Long operational pulses and short dephasing times due to fast recombination, a consequence of the large dipole moment in 


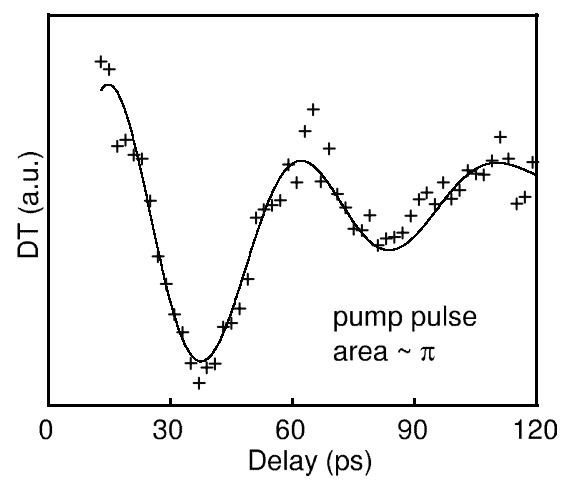

Fig. 3. An entangled state involving two polarized excitons confined in single dots was created and detected optically as evidenced by quantum beats between states $|01\rangle$ and $|10\rangle$ shown. The quantum coherence time between these two states is directly extracted from the decay of the envelope.

natural QDs, are two key issues that lead to fidelity below one for the gate. The combination of a short operational pulse (1 ps) and long population relaxation times ( $200 \mathrm{ps}$ ) with no pure dephasing would lead to a much improved gate with fidelity, $(F)$, as high as 0.97 with the optimal separation between pulses.

In order to increase the quantum operations beyond one dot, interdot exciton interaction is required. One proposal is to use an electric field to increase the dipole-dipole interaction between two excitons in separate dots. ${ }^{(6)}$ Dynamic control of the electric field means some sacrifice of the clock speed of the optical control. Another proposal is to put the dots in an optical cavity so that the cavity mode can serve as a data bus for the exciton qubits. ${ }^{(36,37)}$

\section{OPTICAL CONTROL OF SPIN QUBITS IN QUANTUM DOTS}

In this section we describe a scheme for a quantum computer that can be scaled up to many qubits based on optical control of the electron spin. The system consists of a 2-dimensional array of self-assembled quantum dots in a plane normal to the growth axis of an epitaxial cake of III-V semiconductors. Each dot is charged with one electron whose two spin states in the lowest orbital serve as the fundamental qubit.

A set of qubit operations required for universal quantum computation (consisting of arbitrary single qubit rotations and a suitable conditional two-qubit operation) are proposed using coherent optical control of 
off-resonance Raman processes which involve the spin states in a static magnetic field transverse to the growth direction. Since the shortest dotcenter to dot-center distance will be tens of nanometers, current nearfield optics capability is unable to address two neighboring qubits. To circumvent this problem, we have developed the zip-code concept of light covering an area containing a dozen or so of dots. Within each zip-code, frequency selection is used to carry out the quantum operations on one or two desired spins. The unintended dynamics of the other spins is eliminated by laser pulse shaping.

We classify the errors in quantum operations into two classes: the unintended dynamics due to the discrete states in the dots and the decoherence effects due to the coupling of the spin degrees of freedom with the environment defined as any system with a continuum of degrees of freedom such as the electromagnetic field and the solid vibrations. The direct spin dephasing time is long because of the weak spin flip terms. The optical decoherence incurred by the optical processes, potentially the strongest component of decoherence, is minimized by keeping the Raman processes off-resonance. The advantage of the optical control is then the fast (subnanosecond) clock speed in relation to very long spin dephasing time (possibly close to milliseconds).

\subsection{Qubit Initialization}

The system needs to be prepared initially to a specific state, say, all spins down. The qubit initialization is to be carried out by first splitting the two spin states in each dot with a constant magnetic field and then optical pumping of the spin up state to the trion state allowing time for relaxation into the spin down states. ${ }^{(38,39)}$

\subsection{Measurement}

Measurement can be carried out through optical recycling transitions between a specific electron spin state and the corresponding trion state under resonant optical excitation. The transition will occur only if the electron is in the targeted spin state. A photon will be emitted after each cycle until there is a spin flip process of the trion or electron, providing a burst of photons. ${ }^{(38)}$

\subsection{Arbitrary One-qubit Operations}

The use of coherent circularly polarized light propagating along the growth axis of the semiconductor layers (denoted as the $z$-axis) for the 
Raman process shown in Fig. 1(d) can produce a single qubit rotation only about the $z$-axis. We have designed a method for a rotation about any axis through any angle by utilizing a static magnetic field pointing away from the $z$-axis and with sufficient Zeeman splitting so that two pulses of different frequencies can separately address the optical excitation (lowest energy trion) of each spin state without interfering with the other state. (22) The three parameters of an arbitrary rotation, such as the Euler parameters or the orientation of rotation axis relative to the magnetic field and the angle of rotation are determined by the relative phase and Rabi frequencies of the two applied pulses connecting the two spin states to the trion state and their common detuning from the trion state.

While we make use of the off-resonance Raman process in the adiabatic regime, our pulse design is conceptually from that for the population transfer using stimulated Raman adiabatic passage (STIRAP). ${ }^{(40)}$ While both methods involve coherent control of the three-level system, STIRAP uses the dark optical dressed state to affect the population transfer whereas we use the other two dressed states to make a spin rotation. The "counter-intuitive" sequence of two pulses used in STIRAP requires the foreknowledge of the initial and the final state, which cannot be used as a qubit operation in quantum computing. Our design for a qubit operation makes use of the remaining two states and works for an arbitrary initial spin state.

\subsection{Two-qubit Logic Gate}

The principle is based on Ref. 19, optically creating a Heisenberg interaction between two spins in two nearest neighbor dots as illustrated in Fig. 4(a). The amount of rotation between the two antiparallel spin states shown in Fig. 4(b) can be controlled by the time duration of the optical pulse. For example, an effective $\pi / 2$ pulse yields a qubit swap. However, to reduce the addressing problem because of the current focusing limit of the optical field, the intermediate state of the excited trion should be chosen to be the common bound states of the two dots. The dots are designed with the ground states of the electrons in different dots isolated from one another but with the first or higher excited states having overlapping wave functions between the nearest neighbors as shown in Fig. 4(b). Thus, for the single-qubit operation, the lowest trion used for the intermediate Raman state is isolated in a dot, whereas for the twoqubit operation, the excited trion state is designed to cover two and only two neighboring dots. 
(a)

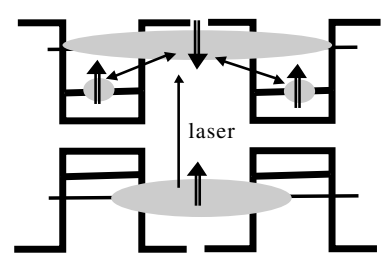

(b)

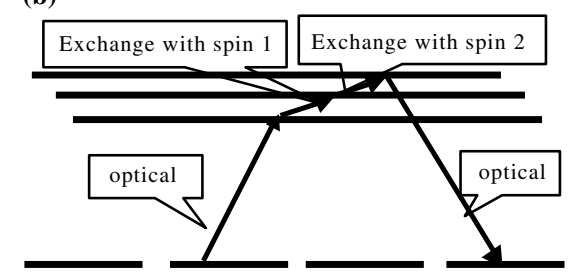

Fig. 4. Optical control of two spins in two separate dots. (a) The single particle energy level scheme; (b) the same process in terms of the four states of the two spins and the excited states with the addition of an exciton.

The excited trion energies of the two dots are brought to coincidence by the dynamic Stark shift (second-order ac electric field induced energy change) while the relevant energy levels from the other dots are kept away from the optical frequencies used. Under ideal operation conditions, the detuning from the intended intermediate state is always less than the energy differences between the intermediate state and other states. To refine the operation for less than ideal circumstances, we propose pulse shaping, to be discussed in Sec. 5.5.

\subsection{The Possibility in Principle of Building a Large Quantum Computer}

The problem of addressing specific single or two qubits belongs to the realm of the avoidance of unintended dynamics. Its solution is key to both specific quantum operations and to the scalability of the computer. In NMR, ${ }^{(41)}$ a sequence of pulses in time is used to accomplish an operation. In quantum optics, ${ }^{(42)}$ a pulse is shaped in the frequency domain to acquire a certain shape in the time domain without drastically increasing the operation time. The pulse shaping approach for quantum operations ${ }^{(7,43,44)}$ in frequency space uses a broader bandwidth than the interdot and intradot energy differences to gain a short operation time. The idea is to use the flexibility of the pulses to return the unintended dynamics to a net zero at the end of the operations. 
The use of the frequency space avoids the time consumed by pulse sequencing in NMR depending on the principle of spin echo, which the dot system cannot afford because of its much shorter decoherence time. The energy difference for unintended dynamics to arise is $0.1-1 \%$ of the laser bandwidth, one to two orders of magnitudes smaller than the NMR design. Our approach does not so much eliminate the unintended dynamics by interference to render the undesired level dark as to bring the recalcitrant electron back to its original state at the end of the pulse. It is possible to limit the overhead of pulse shaping to have a power-law dependence on the number of qubits. ${ }^{(7)}$ We have simulated a few pulse-shaping operations to study their performance quality ${ }^{(7,43)}$ but much work for the spin operations remains to be carried out.

\subsection{Decoherence}

There are two sources of decoherence. One is spin relaxation whose time is long, as discussed above, although the transverse relaxation time $\mathrm{T}_{2}$ in a single dot has yet to be measured. The decoherence due to the optical processes during the operations involving the additional excitons could be fast and has to be ameliorated by design, such as detuning or pulse shaping.

\section{SUMMARY}

The experience of building quantum logic devices based on optically driven quantum dots has provided the basis for moving from the exciton qubit to the spin qubit. Many benchmark features predicted for zerodimensional systems have been readily observable in the model system of natural dots formed by interface fluctuations once the technical challenges of nano-optical probing to enable the study of individual quantum dots were overcome. The body of work has shown that the quantum dots do indeed have the optical features of sharp energy level structure associated with atomic systems and that decoherence effects that are associated with the continuum states of higher-dimensional structures are not an issue in these systems, in general. Multiple reports of exciton Rabi oscillations demonstrated on different types of quantum dots verify the robustness of such qubit rotational operations. Evaluation of the performance of the these systems show surprisingly high fidelity for initial studies, and further progress in materials development and the application of optical pulse shaping and coherent control techniques will surely result in considerable improvement. 
While further development of exciton based qubit systems will undoubtedly lead to impressive performance, much of the future work will be focused on extending the exciton system to the $\Lambda$ system of Fig. 1(d) by doping a QD with a single electron. Moving to the spin based qubit will lead to longer coherence times with the promise of improved performance as aimed at quantum computing. The challenges here lie in developing further control of materials fabrication, which will be paired with more sophisticated schemes for optical excitation and coherent control. Full characterization of the density matrix will be essential for complete understanding of the system, and means for reliable readout and initialization will need to be experimentally investigated.

Advances in materials work combined with new optical probing and coherent optical control developments have enabled surprisingly fast development of optically driven quantum dot systems for quantum information processing. Innovative proposals by many groups have identified several approaches now to extend this work in a scalable manner. ${ }^{(18-20,24,36,37)}$ Many critical questions remain, however, and further work is required in all areas.

\section{ACKNOWLEDGMENTS}

We thank the numerous coauthors of our papers in the references for their essential contributions and acknowledge with gratitude the financial support of ARDA/NSA/ARO, DARPA, ONR, NSF and FOCUS.

\section{REFERENCES}

1. A. Barenco, D. Deutsch, A. Ekert, and R. Jozsa, Phys. Rev. Lett. 74, 4083 (1995).

2. D. Gammon, E. S. Snow, B. V. Shanabrook, D. S. Katzer, and D. Park, Phys. Rev. Lett. 76, 3005 (1996).

3. D. Gammon, E. S. Snow, B. V. Shanabrook, D. S. Katzer, and D. Park, Science 273, 87 (1996).

4. D. Gammon and D. G. Steel, Phys. Today 55(10), 36 (2002).

5. F. Troiani, U. Hohenester, and E. Molinari, Phys. Rev. B 62, 2263 (2000).

6. E. Biolatti, R. C. Iotti, P. Zanardi, and F. Rossi, Phys. Rev. Lett. 85, 5647 (2000).

7. P. Chen, C. Piermarocchi, and L. J. Sham, Phys. Rev. Lett. 87, 067401 (2001).

8. N. Bonadeo, G. Chen, D. Gammon, D. Park, D. S. Katzer, and D. G. Steel, Phys. Rev. Lett. 83, 2267 (1998).

9. N. Bonadeo, J. Erland, D. Gammon, D. Park, D. S. Katzer, and D. G. Steel, Science 282, 1473 (1998).

10. T. H. Stievater, X. Li, D. G. Steel, D. Gammon, D. Park, D. S. Katzer, C. Piermarocchi, and L. J. Sham, Phys. Rev. Lett. 87, 133603 (2001).

11. H. Kamada, H. Gotoh, J. Temmyo, T. Takagahara, and H. Ando, Phys. Rev. Lett. 87, 246401 (2001). 
12. H. Htoon, T. Takagahara, D. Kulik, O. Baklenov, A. L. Holmes, Jr., and C. K. Shih, Phys. Rev. Lett. 88, 087401 (2002).

13. A. Zrenner, E. Beham, S. Stufler, F. Findeis, M. Bichler, and G. Abstreiter, Nature 418, 612 (2002).

14. G. Chen, N. H. Bonadeo, D. G. Steel, D. Gammon, D. S. Katzer, D. Park, and L. J. Sham, Science 289, 1906 (2000).

15. G. Chen, T. H. Stievater, E. T. Batteh, X. Li, D. G. Steel, D. Gammon, D. S. Katzer, D. Park, and L. J. Sham, Phys. Rev. Lett. 88, 117901 (2002).

16. X. Li, Y. Wu, D. G. Steel, D. Gammon, and L. J. Sham, Submitted.

17. X. Li, Y. Wu, D. G. Steel, D. Gammon, T. H. Stievater, D. S. Katzer, D. Park, C. Piermarocchi, and L. J. Sham, Science 301, 809 (2003).

18. D. Loss and D. P. Dvincenzo, Phys. Rev. A 57, 120 (1998).

19. C. Piermarocchi, P. Chen, L. J. Sham, and D. G. Steel, Phys. Rev. Lett. 89, 167402 (2002).

20. E. Pazy, E. Biolatti, T. Calarco, I. Damico, P. Zanardi, F. Rossi, and P. Zoller, Europhys. Lett. 62, 175 (2003); A. Imamoglu, E. Knill, L. Tian, and P. Zoller, Phys. Rev. Lett. 91, 017402 (2003); P. Solinas, P. Zanardi, N. Zanghi, and F. Rossi, Phys. Rev. B 67, 121307 (2003); F. Troiani, E. Molinari, and U. Hohenester, Phys. Rev. Lett. 90, 206802 (2003); T. Calarco, A. Datta, P. Fedichev, E. Pazy, and P. Zoller, Phys. Rev. A 68, 12310 (2003).

21. R. J. Warburton, C. Schaflein, D. Haft, F. Bickel, A. Lorke, K. Karrai, J. M. Garcia, W. Schoenfeld, and P. M. Petroff, Nature 405, 926 (2001).

22. P. Chen, C. Piermarocchi, L. J. Sham, D. Gammon, and D. G. Steel, Phys. Rev. B 69, 075320 (2004).

23. J. G. Tischler, A. S. Bracker, D. Gammon, and D. Park, Phys. Rev. B 66, 81310R (2002).

24. D. P. DiVincenzo, in Semiconductor Spintronics and Quantum Computation, D. D. Awschalom, D. Loss, and N. Samarth (Eds), (Springer, Berlin, 2002), p. 221.

25. D. Birkedal, K. Leosson, and J. M. Hvam, Phys. Rev. Lett. 87, 227401 (2001).

26. P. Borri, W. Langbein, S. Schneider, U. Woggon, R. L. Sellin, D. Ouyang, and D. Bimberg, Phys. Rev. Lett. 87, 157401 (2001).

27. M. Bayer and A. Forchel, Phys. Rev. B 65, 041308 (2002).

28. R. Hanson, B. Witkamp, L. M. K. Vandersypen, L. H. Willems van Beveren, J. M. Elzerman, and L. P. Kouwenhoven, Phys. Rev. Lett. 91, 196802 (2003).

29. Ishikawa, T. Nishimura, S. Kohmoto, K. Asakawa, Appl. Phys. Lett. 76, 167 (2000).

30. M. Bayer, P. Hawrylak, K. Hinzer, S. Fafard, M. Korkusinski, Z. R. Wasilewski, O. Stern, and A. Forchel, Science 291, 451 (2001).

31. J. R. Guest, T. H. Stievater, G. Chen, E. T. Tabak, B. G. Orr, D. G. Steel, D. Gammon, and D. S. Katzer, Science 293, 2224 (2001).

32. Q. Wu, D. Grober, D. Gammon, and D. S. Katzer, Phys. Rev. Lett. 83, 2652 (1999).

33. K. Matsuda, T. Saiki, S. Nomura, M. Mihara, Y. Aoyagi, S. Nair, and T. Takagahara, Phys. Rev. Lett. 91, 177401 (2003).

34. A. Lenihan, M. V. Gurudev Dutt, D. G. Steel, S. Ghosh, and P. K. Bhattacharya, Phys. Rev. Lett. 88, 223601 (2002).

35. J. F. Poyatos, J. I. Cirac, and P. Zoller, Phys. Rev. Lett. 78, 390 (1997).

36. A. Imamoglu, D. D. Awschalom, G. Burkard, D. P. Divincenzo, D. Loss, M. Sherwin, and A. Small, Phys. Rev. Lett. 83, 4204 (1999).

37. T. A. Brun and H. Wang, Phys. Rev. A 61, 032307 (2000).

38. A. Shabaev, A. L. Efros, D. Gammon, and I. A. Merkulov, Phys. Rev. B 68, 201305 (2003).

39. W. Yao, R. Liu, and L. J. Sham, cond-mat/0312272.

40. K. Bergmann, H. Theuer, and B. W. Shore, Rev. Mod. Phys. 70, 1003 (1998). 
41. M. H. Levitt, Prog. NMR Spect. 18, 61-122 (1986).

42. A. M. Wiener, Prog. Quant. Electr. 19, 161-237 (1995).

43. C. Piermarocchi, P. Chen, Y. Dale, and L. J. Sham, Phys. Rev. B 65, 075307 (2002).

44. D. Goswami, Phys. Rep. 374, 385 (2003). 